\title{
Integración de las TIC para una "buena enseñanza": opiniones, actitudes y creencias de los docentes en un instituto de formación de formadores
}

\section{Integrating ICT for 'good educational practices': opinions, attitudes and beliefs of teachers in a Teacher Training Institute}

Diego Rombys-Estévez

\section{Resumen}

El presente estudio aborda las opiniones, actitudes y creencias de los docentes de un instituto de formación magisterial acerca de la integración de las TIC en la educación.

La investigación corresponde a un enfoque cualitativo descriptivo que emplea varias técnicas asociadas, en general a un estudio de caso. Entre ellas destacamos como herramienta principal el uso de entrevistas, incluyendo el empleo de imágenes para evocar recuerdos y sentimientos, así como técnicas complementarias que incluyen un cuestionario. A partir del análisis de la literatura se perfeccionaron dichos instrumentos $\mathrm{y}$, posteriormente, se triangularon dichas técnicas.

Entre los principales hallazgos de esta investigación se constató que quienes presentan enfoques educativos centrados en la enseñanza tienden a considerar de forma más crítica a las TIC. Complementariamente, se identificaron otros aspectos como, por ejemplo, que quienes están dispuestos a aprender acerca del uso de las TIC "con y de los alumnos" presentan actitudes más proclives a incorporar estas tecnologías que quienes creen que ello significa una pérdida de autoridad y afecta su autoestima. Algo similar ocurre en relación a cómo consideran que las TIC afectan al empleo tradicional de los libros.

En las reflexiones finales se incluyen sugerencias para enfrentar con mayores posibilidades estos desafíos, y construir adecuados equilibrios a partir del aporte de los docentes a los efectos de contemplar las diferentes visiones que hacen a una "buena enseñanza".

En cuanto a la oportunidad de esta investigación, basta mencionar que en virtud del Plan CEIBAL en los próximos años irán llegando progresivamente a las instituciones de formación las primeras generaciones "criollas" de "nativos digitales" para formarse como maestros. En dicho marco esperamos que puedan desarrollarse como docentes sin barreras conceptuales que limiten sus oportunidades y las de sus futuros alumnos.

PALABRAS CLAVE: TIC, integración de TIC en la educación, actitudes y creencias docentes, buena enseñanza, pensamiento docente, formación docente

\section{Abstract}

This research focuses on the opinions, attitudes and beliefs of teachers at a teacher training institute, about the integration of ITCs in their teaching practice.

The research develops a qualitative and descriptive approach, involving a variety of techniques applied to a case study. Among these techniques, the conduction of interviews is highlighted, which included the use of images in order to evoke memories and feelings. Also a questionnaire was implemented as a complementary tool. Based on the study of specialized bibliography, these research tools were enhanced and the results were triangulated.

Among the main findings of this project, it was noticed that those who practice a teachercentered approach tend to be more critical of ITCs. Additionally, teachers who were eager to 
learn about the use of ITCs "with and from the students" were also more willing to include the new technology in their teaching than others who considered that this fact would mean a loss in terms of authority and self esteem. Something similar happened related to the way teachers feel about how ITCs affect the traditional use of textbooks.

There are some final considerations on how to face these challenges and build an adequate balance among the different views on teaching in order to promote good practices.

The context of this research is given by the fact that as a result of the implementation of the so-called "Plan CEIBAL in Uruguay, the new generations of local "digital natives", more competent in the use of computer technology, will progressively enter Teacher Training Institutes. It is desirable that their teaching careers will not be held up by concept barriers which may limit their chances as well as those of their students.

KEY WORDS: ITCs Technology Integration, teachers attitudes and beliefs, good practices, Teachers thinking, teachers training

\section{Introducción}

El problema se inscribe en las líneas de investigación que abordan el debate sobre el valor de las tecnologías de información y comunicaciones (TIC) como tecnologías educativas, contribuyente a definir los motivos y contextos que justifiquen y promuevan su incorporación en la actividad docente.

Se busca dar respuestas contextualizadas a los desafíos que la sociedad contemporánea plantea a la educación, en particular mejorando la pertinencia y relevancia de los aprendizajes. Partimos para ello de la búsqueda y comprensión de los significados que los docentes atribuyen a estas tecnologías, y en particular a lo que entienden debe ser, en nuestro contexto, una "buena enseñanza".

Se trata entonces de conocer y comprender las opiniones, actitudes y creencias de los docentes, de un Instituto de formación magisterial respecto a la integración de las TIC a la educación en general y a su práctica docente en particular, lo cual nos lleva a la definición de los siguientes objetivos:

Identificar opiniones, actitudes y creencias de los docentes del instituto respecto a la integración educativa de las TIC, y en particular en relación con lo que consideran una "buena enseñanza".

Distinguir y categorizar a partir de los significados emergentes, posibles factores que faciliten o limiten, favorezcan u obstaculicen la integración de las TIC en las prácticas educativas de los docentes a nivel del instituto de formación considerado.

En suma, se aspira a lograr un mejor conocimiento y comprensión de los procesos que intervienen en la integración educativa de las TIC posicionándonos desde el punto de vista del docente, buscando generar aportes que favorezcan el desarrollo de una "buena enseñanza". Nos aproximaremos a la complejidad del tema a partir de las consideraciones de los propios actores, cuya subjetividad y significado se tratará de recuperar indagando las razones que argumentan, y las emociones que generan las TIC en el colectivo estudiado.

En cuanto al contexto y oportunidad de la investigación, cabe mencionar que pese a los avances respecto a la incorporación de la tecnología en la educación, aun los países más desarrollados que han superando problemas de capacitación y acceso se considera que existen desafíos y promesas incumplidas (Buckingham 2008, Area Moreira 2010, Boza 2010 entre otros). Se identificaron obstáculos adicionales, los que han sido denominados como barreras o factores internos asociados a las creencias de los docentes (Ertmer, 1999, 2005) siendo consideradas como la "frontera final" para una adecuada incorporación de las TIC en la educación. 
A nivel nacional, el desarrollo progresivo del Plan CEIBAL (2008) sitúa a nuestro objeto de estudio sobre los principales ejes de atención académica y a la elección de un instituto de formación docente como una oportunidad para contribuir a la mejor comprensión de estos aspectos. En particular, se buscó acceder a una institución donde la amplitud del universo a considerar permitiera el empleo de técnicas cualitativas de forma significativa.

\section{Antecedentes y fundamentación teórica}

\subsection{La perspectiva de la enseñanza}

Investigaciones recientes ubican a las creencias y concepciones de los docentes acerca de los procesos de enseñanza y de aprendizaje como elementos centrales de las líneas de investigación que tratan el denominado "Pensamiento del profesor" (Serrano Sánchez, 2010). A fines de la década de los 80, Pérez Gómez y Gimeno Sacristán manifestaban que: "A lo largo de esta fecunda década de investigaciones sobre los procesos de pensamiento del profesor aparece gradualmente con más claridad el hecho de que los juicios, decisiones y propuestas que hace el profesor se derivan de su peculiar modo de interpretar su experiencia" $(1988,44)$. A comienzos de la década de 1990 se fue desarrollando el análisis de la toma de decisiones de los profesores a partir de una perspectiva centrada en lo cognitivo, generando diferentes conceptos. Se fueron constituyendo así nuevas líneas de investigación catalogadas como "conocimiento profesional de los profesores" procurando una profundización en el estudio de los contenidos que enseñan (Marcelo, 1992).

$\mathrm{Al}$ ir evolucionando, las teorías relacionadas al pensamiento y conocimiento de los docentes fueron incorporando las perspectivas disciplinares, a la vez que se involucraban con investigaciones centradas en la formación

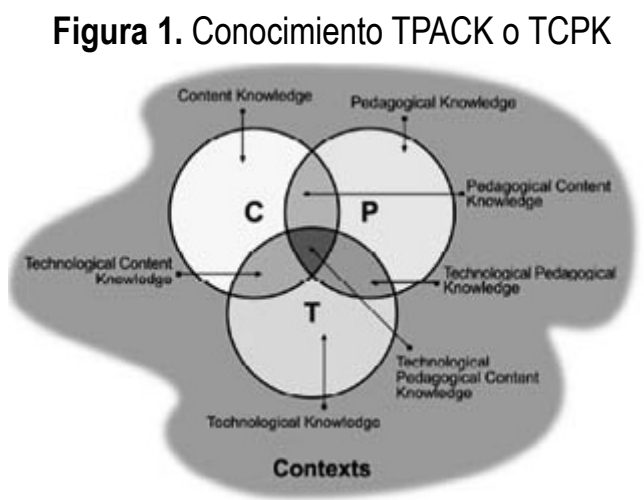

Tomado de Mishra y Koehler $(2008,3)$ docente. Se fue constituyendo así la base de los programas orientados a la formación inicial de profesores, a los profesores principiantes y a los programas para el desarrollo profesional docente (Marcelo, 2001). En dicho contexto, ha cobrado creciente importancia la integración de las TIC a la educación.

Si bien el "conocimiento didáctico del contenido" plantea una óptica desde la cual se puede considerar dicha integración, se fueron proponiendo progresivamente varios modelos que específicamente relacionaron al "conocimiento pedagógico" y al "conocimiento de contenidos" con la tecnología. La aceptación internacional de este enfoque se consolida con la difusión de la propuesta de Mishra y Koehler a través de la cual consideran que este triple relacionamiento (Fig.1) constituye el camino más efectivo para integrar a las TIC en la educación.

Actualmente se considera que la formación docente es un proceso integrado al desarrollo profesional que se extiende a lo largo de la vida (Ávalos, 2007; Vaillant, 2007). La formación inicial del docente se consolida y proyecta en las siguientes etapas de su carrera a partir de las experiencias profesionales y de las instancias educativas de las que participa. Incluso, se puede considerar que en el proceso de formación los puntos de vista de los docentes se construyen a partir de las creencias que traen consigo y sobre las cuales influyen sus experiencias académicas y prácticas (Vaillant, 2007:209). 
Una vez finalizada la etapa de formación inicial, Ávalos identifica dos grandes estrategias de formación: por un lado las que denomina como "cercanas a la capacitación" y en el otro extremo, las asociadas a "talleres reflexivos" y "desarrollo profesional autónomo" (2007:81) posiciones que lejos de ser excluyentes enmarcan múltiples variantes que, coexistiendo en diferente grado, conforman los sistemas de formación continua.

Estos antecedentes, entre otros, marcan una evolución de los modelos o teorías del "pensamiento del profesor" hacia paradigmas asociados al conocimiento al "aprender a enseñar", y al desarrollo profesional docente manteniendo las creencias de los educadores entre los ejes principales de estas líneas de investigación.

\subsection{Opiniones, actitudes y creencias de los docentes en el marco de la "buena enseñanza"}

Del relevamiento bibliográfico y los respectivos hallazgos más significativos de la literatura especializada en esta temática (Fig.2) se arribó a las siguientes definiciones operativas:

\section{Buena enseñanza}

Es la actividad educativa eficaz y reflexiva que incluye, pero excede, lo puramente instrumental y cognitivo (Burbules y Callister, 2001), adquiriendo un carácter profundamente moral (Litwin, 2005). Implica por lo tanto una dimensión epistemológica derivada de lo disciplinar y una dimensión ético-moral.

\begin{tabular}{|c|c|c|}
\hline Creencia & Actitud & Opinión \\
\hline $\begin{array}{l}\text { Proposiciones, premisas que } \\
\text { mantienen las personas acerca } \\
\text { de lo que consideran verdadero } \\
\text { (Richardson, } 1996 \text { en Marcelo, } \\
\text { 5001:37), que implican juicios } \\
\text { y connotaciones afectivas, } \\
\text { que en general orientan sus } \\
\text { comportamientos y actitudes } \\
\text { (Pajares, 1992) y que se van } \\
\text { construyendo influidas por el } \\
\text { conocimiento y experiencias } \\
\text { personales (Marcelo, 2001). }\end{array}$ & $\begin{array}{l}\text { Predisposición de la persona } \\
\text { a responder favorable o } \\
\text { desfavorablemente a un objeto, } \\
\text { persona o evento (Ajzen, } \\
\text { 2001), potencialmente influida } \\
\text { u orientada por sus creencias } \\
\text { (Van Braak y Tandeur, 2009) y } \\
\text { reflejada a través de las acciones } \\
\text { que realiza o las intenciones u } \\
\text { opiniones que declara (Sarabia, } \\
\text { 1992). }\end{array}$ & $\begin{array}{l}\text { Manifestación verbal } \\
\text { de las actitudes que } \\
\text { expresan la posición de } \\
\text { la persona respecto al } \\
\text { objeto considerado, ya } \\
\text { sea emitiendo un juicio } \\
\text { de valor o considerando el } \\
\text { efecto futuro (predictivo) } \\
\text { de la situación (Sarabia, } \\
\text { 1992), la cual corresponde } \\
\text { a la posición que la } \\
\text { persona toma frente a } \\
\text { cuestiones de relevancia } \\
\text { social. (Araya Umaña, } \\
\text { 2002). }\end{array}$ \\
\hline
\end{tabular}

\subsection{TIC y educación}

\section{Figura 2}

Otro capítulo relacionado a los fundamentos de la investigación lo constituye la consideración del rol de las TIC a partir de lo que inicialmente se denominó "Sociedad de la Información" y su proyección hacia la educación. Conceptos como "brecha digital", "nativos e inmigrantes digitales" (Prensky, 2001) son especialmente considerados en las investigaciones educativas en las que se aborda la relación entre educación y las nuevas tecnologías.

A nivel nacional, si bien la incorporación de las TIC en la educación avanzaba de forma paulatina, es a partir del Plan CEIBAL (2008) que toman un rol protagónico en la consideración de los docentes, generando diversos tipos de reacciones que influyen en sus puntos de vista respecto a las TIC. 
En suma, la integración de las TIC en la educación forma parte del marco teórico de esta investigación, en el cual es necesario considerar los estándares internacionales (Unesco, 2008; Vaillant,2005, Area Moreira, 2010) e incluso la integración de estos temas en la formación de maestros en el Uruguay (ANEP, 2001; Rodríguez Zidán y Téliz, 2001, entre otros).

\subsection{Antecedentes de investigaciones relacionadas al objeto de estudio}

Varias investigaciones realizadas en países desarrollados exploraron la relación entre las creencias de los docentes y el uso de computadoras en el aula. Este tipo de estudio revisten un doble interés.

El siguiente cuadro (Fig.3) muestra un resumen de las principales referencias relevadas, incluyendo antecedentes locales.

\begin{tabular}{|l|l|}
\hline \multicolumn{1}{|c|}{ Investigación } & \multicolumn{1}{|c|}{ Aspectos referenciados } \\
\hline $\begin{array}{l}\text { Ertmer (1999 y 2005) } \\
\text { EE.UU. }\end{array}$ & $\begin{array}{l}\text { Identificación y clasificación de las barreras que dificultan la } \\
\text { integración de TIC en la educación }\end{array}$ \\
\hline Bai y Ertmer (2008) EE.UU. & $\begin{array}{l}\text { Relación entre el uso de TIC y las actitudes de los docentes } \\
\text { en formación, para lo cual recomiendan el uso de técnicas } \\
\text { cualitativas. }\end{array}$ \\
\hline $\begin{array}{l}\text { Tondeur y van Braak (2008) } \\
\text { Bélgica }\end{array}$ & $\begin{array}{l}\text { Cuestionario estructurado para determinar relación de los } \\
\text { enfoques educativos y el uso de TIC }\end{array}$ \\
\hline $\begin{array}{l}\text { Schmidt, Baran, Thompson, } \\
\text { Mishra, Koehler y Shin } \\
\text { (2009) EE.UU. }\end{array}$ & $\begin{array}{l}\text { Desarrollo y validación de un cuestionario de auto evaluación } \\
\text { para medir conocimientos pedagógicos, de contenido y/o } \\
\text { tecnología y sus relaciones (TPACK) }\end{array}$ \\
\hline $\begin{array}{l}\text { Area Moreira (2010) } \\
\text { España }\end{array}$ & $\begin{array}{l}\text { Integración y uso pedagógico de las TIC en centros educativos } \\
\text { (Proyecto Medusa) considerando las perspectivas del } \\
\text { profesorado hacia las TIC empleando cuestionarios /o entrevistas }\end{array}$ \\
\hline $\begin{array}{l}\text { Boza, Tirado y Guzmán- } \\
\text { Franco (2010) España }\end{array}$ & $\begin{array}{l}\text { Influencia de la opinión del profesorado sobre la tecnología en } \\
\text { su aprovechamiento didáctico e integración curricular de las TIC } \\
\text { en los centros docentes empleando cuestionarios }\end{array}$ \\
\hline $\begin{array}{l}\text { Rodríguez Zidán, Ferreira } \\
\text { Cabrera, Téliz (2011) } \\
\text { Uruguay }\end{array}$ & $\begin{array}{l}\text { Opiniones y percepciones docentes sobre el cambio y la gestión } \\
\text { escolares en las escuelas públicas del departamento de Salto, } \\
\text { Uruguay empleando encuestas y entrevistas }\end{array}$ \\
\hline
\end{tabular}

Figura 3

Reviste especial interés la literatura que específicamente trata el corte entre la incorporación de tecnología y conceptos asociados a una "buena enseñanza".

Actualmente existe una visión bastante extendida en cuanto a que: "el tema en cuestión ya no es si los docentes deben integrar las TIC en sus prácticas, sino cómo transformar su enseñanza a través de la tecnología para crear nuevas oportunidades de aprendizaje". (Angeli y Valanides, 2009:167).

El conocimiento del docente en materia pedagógica, de contenidos y tecnología constituye en su conjunto la base de las "buenas prácticas" y la "buena enseñanza", aspecto que comparten Schmidt, Baran, Thompson, Mishra, Koehler y Shin (2009). Por su parte, Ertmer y OttenbreitLeftwich (2010) son más terminantes al afirmar que hace veinte años un docente podía pensar que estaba haciendo un buen trabajo, aun cuando sus estudiantes no usaran tecnología. 
Por nuestras latitudes, si bien también se afirma que actualmente no es posible concebir una educación de calidad sin la integración de las TIC en las aulas (Lugo en Gairín Sallán, et al., 2011, entre otros) aún son limitadas las menciones académicas que las asocian directamente a la "buena enseñanza", al conocimiento integral de los docentes en materia pedagógica, de contenidos y tecnología, y a los enfoques centrados en los logros de los aprendizajes de los alumnos.

\section{Metodología}

El enfoque de esta investigación es claramente cualitativo, al grado que es difícil visualizar una problemática asociada a las TIC cuyas características requieran con mayor énfasis tal aproximación. De los modelos existentes consideramos especialmente aplicable el estudio de caso el cual, según Stake, busca indagar: "la particularidad y la complejidad de un caso singular, para llegar a comprender su actividad en circunstancias importantes" $(1998,11)$, (1998:11) en nuestro caso tomando como referencia el rol de "intérprete" que el mencionado autor le asigna al investigador (ibidem, 20).

\subsection{Caracterización de las técnicas empleadas}

La consideración del objeto de estudio nos llevó a seleccionar a la entrevista como elemento central de la indagación, en particular como forma de tratar de interpretar las posibles creencias asociadas a la subjetividad del pensamiento docente. Para poder aproximarnos progresivamente a la realidad de la institución y así contextualizar adecuadamente las demás técnicas, se optó por realizar entrevistas exploratorias con autoridades referentes de la institución y observar los aspectos principales que sostienen y regulan las actividades asociadas a las TIC, incluyendo la consideración de los programas de estudio vigentes para Magisterio (ANEP,2008).

Otro elemento de contextualización previo a las entrevistas lo constituyó el empleo de un cuestionario anónimo, auto-administrado y semi-estructurado, a través del cual se buscó obtener datos para el posterior análisis y triangulación de resultados, así como una forma introductoria de invitar y motivar a los docentes a participar de las entrevistas. El cuestionario fue elaborado en base a las dimensiones que se determinaron del marco teórico, conteniendo 18 preguntas estructuradas relativas al perfil y 21 preguntas acerca de los temas en cuestión. En su mayoría se emplearon escalas tipo Likert para actitudes tomando como base general los conceptos de Morales Vallejo (2011), las que fueron perfeccionadas por medio del empleo de cuestionarios de prueba.

En cuanto a las entrevistas, las preguntas cubrieron los siguientes aspectos:

Dimensiones que definen una buena enseñanza ;

El aula y sus interacciones, proyección a veinte años

Comentarios que le merecen imágenes asociadas a las TIC

Rol de las TIC en el marco de una "buena enseñanza"

Motivaciones y desafíos para la integración de TIC en la educación

El empleo de imágenes (pregunta 3), resultó de gran utilidad. Tomamos como base conceptos de Harper (2002) y Clark- Ibáñez (2004), en los cuales a través de la "foto evocación" se busca provocar un estímulo en el entrevistado en relación al objeto de estudio, apelando a la faz emotiva del docente más que a su racionalidad. El universo de estudio estaba constituido por los 25 docentes del instituto, y se realizaron 13 entrevistas producto de una muestra intencional no probabilística que intentó cubrir las variables que se identificaron como más relevantes. 


\subsection{Análisis y triangulación de técnicas}

Las respuestas del cuestionario se consolidaron en una planilla de cálculo que permitió una primera aproximación cuantitativa a los resultados, su posterior representación gráfica y finalmente la interpretación cualitativa de los mismos. Asimismo se procuró mantener en bloques las preguntas de similar índole, de forma de facilitar el proceso de síntesis que culminó con la determinación de los hallazgos.

Las estrategias de categorización de los datos para el análisis de las entrevistas fueron construidas inicialmente a partir del marco teórico elaborado. Durante el análisis se fueron identificando temáticas y significados emergentes para integrarlos con las categorías existentes (Merriam, 1998) acorde a los objetivos definidos. Se desgrabaron todas las entrevistas tratando de respetar la forma en que se expresaban los docentes, así como se registraron aspectos contextuales que permitieran una mejor interpretación de sus expresiones. Primariamente se realizó el análisis de cada entrevista en forma integral tratando de identificar aspectos no contemplados en la categorización inicial. Este primer análisis fue resumido en una tabla de forma de contar con una visión global de todas las entrevistas. Posteriormente se abordó cada una de las interrogantes considerando las expresiones más significativas de todos los docentes, comparando y categorizando las respuestas, de forma de ir incorporando las citas representativas, compararlas con los restantes resultados e integrarlas en los hallazgos y conclusiones.

Asimismo, al ser aplicadas las mismas preguntas base a todos los entrevistados, se habilitó el cruzamiento de sus respuestas. Los resultados generales del cuestionario docente también permitieron confrontar los resultados con las respuestas abiertas de las entrevistas dado que fueron construidos a tales efectos. Completando la triangulación de técnicas, se consideró el análisis de los contenidos de los principales programas curriculares de la asignatura Informática, así como asignaturas orientadas a la formación pedagógica (Pedagogía y Didáctica) de forma de poder contrastarlos con los resultados de las entrevistas. Finalmente y previo a realizar las conclusiones, los hallazgos fueron confrontados con las referencias tomadas de la bibliografía.

\section{Discusión de los hallazgos}

En este apartado se procura integrar y contrastar los hallazgos derivados de la aplicación de las técnicas empleadas con la literatura consultada, agrupándolos a partir de los aspectos que se consideraron más significativos.

\section{1. "Buena enseñanza", calidad educativa y TIC: según los docentes no siempre "van de la mano"}

A partir del resultado de las entrevistas, podemos señalar que entre los docentes del instituto no existe una visión consensuada de lo que para ellos significa una "buena enseñanza". Incluso fue bastante heterogénea la forma de abordar la respuesta; desde el uso de largas pausas o interjecciones para tratar de articular la idea, hasta quienes con total seguridad marcaron respuestas casi en carácter de "postulado", posible reflejo de sus enfoques pedagógicos: "Es bueno cuando: a; es claro, b; explícito, c; el otro puede dar cuenta de aquello que le transmito..." (D12)

La forma de expresar la respuesta transitó desde proposiciones sintéticas: "Una buena enseñanza tiene que aportar a la calidad y la calidad esteee creo que se logra con una profundización del conocimiento y las formas de enseñar" (D12) hasta exhaustivas referencias y ejemplos descriptivos de múltiples aspectos. Muy pocos docentes mencionaron en las entrevistas la existencia de componentes epistemológicos y ético-morales a la vez. Esta situación contrasta notablemente con los resultados del cuestionario. 
Al ser mostrados los mismos a los docentes como opciones, el 100\% de ellos identificó la existencia de ambos componentes en la "buena enseñanza".

En relación a estos hallazgos, la bibliografía consultada da cuenta de un creciente rol de las TIC en la educación en relación a la "buena enseñanza", al grado de que muchos autores consideran que actualmente no es posible lograr una educación de calidad sin el concurso de las mismas. Si bien estas concepciones son aceptadas por académicos a nivel de Latinoamérica, lo incipiente del desarrollo de las TIC en la región no permite contrastarlas con las manifestaciones de los docentes. Esta limitación lleva a que no sea sorprendente que sólo uno de los docentes entrevistados mencione a las TIC en su definición de "buena enseñanza", pese a que varios manifiestan un alto grado de conocimiento y adhesión a las mismas. Podemos destacar entonces que a nivel del discurso de los docentes, las TIC son un tema ausente en los significados que los docentes atribuyen a la "buena enseñanza".

\subsection{La "buena enseñanza" y los diferentes conocimientos en juego: el discurso omitido}

Los docentes tampoco expusieron ideas que hicieran referencia, incluso de forma velada o implícita, al relacionamiento entre el conocimiento tecnológico con el pedagógico y el de contenidos (TCPK o TPACK por sus siglas en inglés) que según documentan algunos de los autores referidos constituye la base para una adecuada integración de las TIC en la educación. A partir de estas "ausencias" en el discurso de los docentes, parecería entonces que no hay (o no se pudieron evocar) reflexiones sobre la implicancia de estos tres tipos de saberes asociados. Los docentes mencionan que necesitan saber más de tecnología, pero no que requieran saber más acerca de cómo usar las TIC para enseñar mejor y para que los estudiantes también aprendan mejor. De hecho, en las entrevistas manejan los términos capacitación y formación casi como sinónimos, siendo que la primera puede relacionarse más con el dominio instrumental y los aspectos técnicos de las TIC y la segunda con las razones y contextos que hacen a un buen uso pedagógico de la tecnología.

En cuanto al programa de formación magisterial vigente (Plan 2008), se observa una clara complementariedad en las asignaturas del "clásico binomio" Pedagogía y Didáctica. No se constata tal grado de relación con las asignaturas que tratan el conocimiento tecnológico, siendo prácticamente nulas las referencias a ellas en los programas de Pedagogía y Didáctica. Si bien se incluyó recientemente como cambio al Plan 2008 la asignatura "Educación e integración de tecnologías digitales", su desarrollo prioriza la visión de lo tecnológico, quedando camino por recorrer en cuanto a las necesarias reflexiones pedagógicas y didácticas asociadas a la "buena enseñanza" y el rol de las TIC para una educación de calidad, relacionando los tres tipos de conocimiento.

\subsection{Las "interacciones" que pueden generar las TIC: ¿Pérdida o ganancia?}

Entre las preocupaciones recurrentes también pudimos identificar el sentimiento de temor ante la posible pérdida de vínculos afectivos que podría traer aparejado el uso de estas tecnologías. En relación a ello, la forma en que los docentes manifestaban su percepción de las interacciones docente-alumno y de los alumnos entre sí mostraba diferencias claras entre quienes expresaban conocimiento y experiencia en el uso de las TIC en el aula respecto del resto. Mientras los primeros señalaban las múltiples posibilidades de interacción que surgían a partir de la mediación de las TIC, los segundos se referían reiteradamente al peligro de aislarse y comprometer los vínculos afectivos, atribuyendo a las TIC una consecuencia cercana a la "despersonalización". 
También fueron múltiples las referencias a las posibilidades de apoyo entre los docentes y el trabajo en grupo como forma de superar limitaciones personales e incrementar la capacitación en materia de TIC, aspecto coincidente con lo que señala la literatura consultada.

“...he tenido que ... aprender ehhh de la tecnología y aprender en grupo o sea, no solamente yo con la máquina sino el grupo ... donde teníamos dudas en común y donde fuimos esteee tratando de solucionarlas ... (D6).

"Es fund... es muy importante, porque esta persona no se anima al principio, pero ... con la influencia de compañeros estee se empieza a animar... "(D9).

“...el camino que hemos recorrido los profesores con respecto a que hemos tenido que unirnos paraaaaa ayudarnos a .... descifrar y a internalizar en esta nueva tecnología..." (D8).

\subsection{Rol docente a partir de las TIC: actitudes y creencias sobre el cuestionamiento de la autoridad en el aula}

Otro de los temores reiterados fue el relativo a la pérdida o posible compromiso del rol del docente. Considerados estos aspectos en el contexto de las manifestaciones de cada entrevistado, surge que estos puntos de vista estuvieron bastante relacionados con la forma en que cada docente percibe su relación con los alumnos. Aquellos docentes que consideran que es importante aprender acerca de las tecnologías, de y con los alumnos, no mostraron temores ni se refirieron a esta posible pérdida de rol. En contraposición se ubican docentes que no se refieren a esta posibilidad, sino que mencionan aspectos tales como el posible menoscabo de su autoridad e incluso miedo a exponer su ignorancia frente a sus alumnos, teniendo como factor común orientaciones mayormente centradas en la enseñanza. La siguiente cita resume en buena medida el sentimiento de varios docentes respecto a la incorporación de las TIC en sus prácticas: “... nos ponen frente a ... desafíos que tal vez delante de otros no aceptamos resolver y ése es un golpe a nuestra autoestima..." (D12).

En oposición a estos temores, y como señalaron varios de los docentes, pretender saber más que los alumnos en materia de tecnología es casi imposible, a la vez que innecesario. Algunos entrevistados incluso señalaron lo beneficioso de mostrarles que el docente también se equivoca. Concretamente proponen dejar en manos de los alumnos los "detalles" de las tecnologías, aprovechando su carácter de flamantes generaciones de "nativos digitales", reafirmando el carácter colectivo y de construcción permanente que caracteriza a la "Sociedad del conocimiento". Asimismo, este enfoque de "complementariedad" contribuiría a "tender puentes" que nos acerquen a la dirección de avance de los alumnos, los cuales, según considera un docente "... están muy adelantados en eso (refiriéndose al empleo de TIC); ellos van por un lado y muchas veces nosotros los docentes vamos por otro...." (D10).

En relación al rol que visualizan los docentes que se mostraron proclives a la integración de las TIC en la educación, fueron mayoritarias las referencias al concepto de orientador, constatándose en las expresiones una reivindicación del docente como elemento clave de la integración de las TIC, aspecto que va totalmente en línea con lo que expresan los autores de la literatura especializada.

\subsection{Enfoques educativos del docente: aspecto "casi" determinante de sus actitudes ante las TIC}

En cuanto a la orientación de los enfoques educativos que surgen en las entrevistas, se observan menciones que se orientan exclusivamente a la enseñanza y a las actividades de los docentes. Sin embargo, también se constatan en los parlamentos referencias a los alumnos, aunque no siempre responden a las ideas o enfoques que se centran en sus logros, tal cual lo consigna la literatura especializada. 


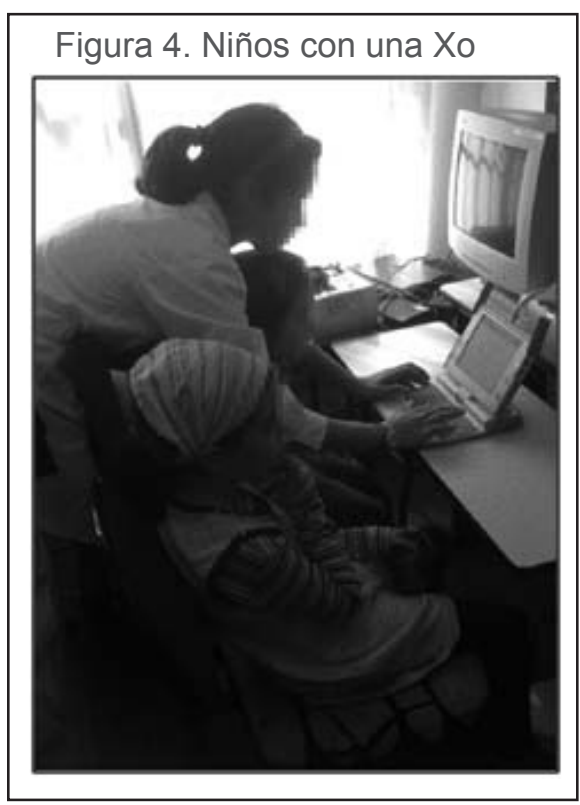

El uso de las imágenes en las entrevistas brindó inesperadamente un elemento que nos permite acercarnos a estos aspectos, dando lugar a un hallazgo muy significativo: todos los docentes que se caracterizaron por hacer referencias mayoritarias a la enseñanza, al observar a niñas de diferentes edades trabajando con la Xo (Fig. 4), describían la escena como una docente con sus alumnas. Por el contrario, quienes mostraron una mayor apertura en sus enfoques, e incluso una mayor predisposición al empleo de TIC, se referían a ellas como niñas de diferentes edades y señalaban la interacción (positiva) que se generaba a partir del uso compartido de la computadora. Entre este último grupo de manifestaciones es reveladora la referencia explícita de dos docentes que indican que los niños pueden aprender de otros niños y que ello es beneficioso para ambos.

"Es hermosa (refiriéndose a la imagen) porque ... acá están, serán tres hermanas ... o será una alumna en una clase ... me recuerda las instancias en primer año cuando los alumnos de sexto y de quinto ... van como tutores a los niños de primero ... y les enseñaron ... (D11); ... les encanta porque se sienten responsables, se sienten.... maestras (se ríe) entonces.... Ellos aprenden más porque fijan, afirman lo que ellos aprendieron..." (D9).

Analizando el resto de las entrevistas de ambos docentes, surge que es una creencia consistente con la forma en la que en general encaran la educación centrada en sus alumnos, mientras que en sentido contrario, estos conceptos están ausentes entre quienes priorizan sus enfoques en la enseñanza y su propia actividad docente.

Respecto a las concepciones pedagógico-didácticas cabe resumir su influencia en la siguiente expresión de un docente: "Y bueno, lo que pasa es que las motivaciones (refiriéndose a la integración de las TIC) dependen de cómo cada uno conciba su disciplina para el desarrollo de la persona, que en último término es eso..." (D7).

\subsection{Tecnologías tradicionales y TIC: ¿Complemento u oposición?}

Retomando la referencia a la forma en que los docentes se refirieron a las distintas tecnologías, se pudieron constatar actitudes disímiles respecto al libro y su relación con las TIC. Los docentes proclives a las TIC mencionaron entre otros conceptos: facilidad de acceso, inmediatez, complementariedad, amplitud y facilidad de búsqueda. En contrapartida, algunos docentes manifestaron temor por la pérdida del hábito de la lectura, las desviaciones en la correcta escritura o incluso pérdida del contacto con el papel. Aquí también existen situaciones que nos llevan a pensar que estas últimas actitudes pueden llegar a ser revertidas, tal cual lo sugiere el caso de un docente que "descubrió" gracias a un buscador de Internet que le mostró un alumno, que los autores y obras que señalaba "existían, estaban allí, cuáles eran sus libros, aparecen todos, cuál es la época, donde es el contexto donde desarrollaron la actividad, es decir, se hicieron presentes en la clase, eso es muy valioso" (D5). Éste y otros ejemplos nos llevan a reflexionar acerca de la utilidad de las clasificaciones de los docentes en cuanto a su actitud hacia las TIC, taxativamente como tecnofílico, tecnofóbico o condicional. 


\subsection{Influencia de la percepción de la competencia de los propios docentes en el manejo de las TIC}

Las respuestas al cuestionario muestran que para ninguno de los docentes las TIC representaban un elemento desconocido; en cambio, al consultarlos acerca de su uso efectivo a nivel administrativo los porcentajes descienden, y luego bajan significativamente al preguntarles si usaban las TIC en forma efectiva en la educación.

La mayor parte de los docentes encuestados (13 en 18, $72 \%$, pregunta 30 del Cuestionario) manifiesta que recibió algún tipo de capacitación técnica relacionada con TIC, porcentaje que disminuye cuando se los consulta en cuanto a formación pedagógica para su uso $(50 \%$, pregunta 31).

Al indagar cómo se sentían respecto al uso de TIC (Fig.5), a medida que la pregunta se acercaba a lo que a nivel del marco teórico tratado se consideran usos de las TIC centrados en los alumnos, los sentimientos de los docentes reflejaban mayor inseguridad, tendencia coincidente con lo que se percibe en las entrevistas donde las referencias a este uso más elaborado de las TIC es mínimo.

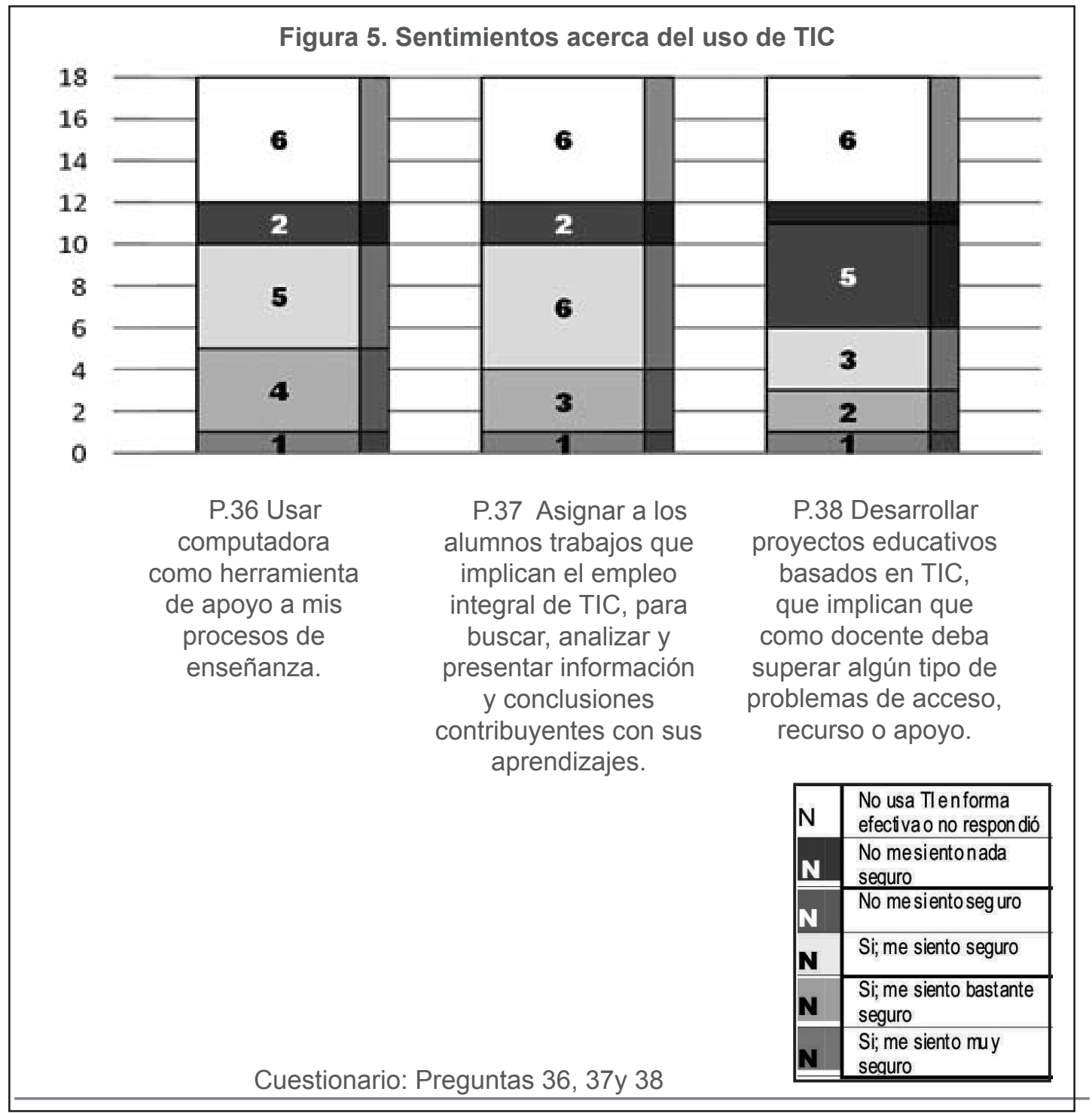

Cuadernos de Investigación Educativa, Vol. 4, № 19, 2013, Montevideo (Uruguay), 69-86. ISSN 1688-9304 79 
"Yo las voy integrando a medida que las voy necesitando...no las integro mucho a la clase esteee ehh porqueeee creo que mi materia que es específicamente $X$ (nombra su asignatura) y la forma como yo la encaro... (menciona el empleo de funciones básicas a nivel administrativo o de preparación de textos para entregar a los alumnos)" (D2).

\subsection{Capacitación en TIC: Condición necesaria pero no suficiente. La relevancia de la Formación "ausente"}

Un elemento bastante común entre quienes presentaron una visión crítica y temores en cuanto a las posibles consecuencias negativas en el uso de las TIC fue que mencionaban explícitamente limitaciones personales en el manejo de las mismas o evidenciaban en sus dichos poco conocimiento tecnológico. La consideración de las entrevistas en forma integral lleva a pensar que el desconocimiento es una de las principales fuentes de actitudes críticas y no a la inversa. Este aspecto también es coincidente con lo que refiere la literatura, más allá de que existen otros elementos que influyen en la integración de las TIC y que estos fenómenos no responden a explicaciones únicas y cartesianas del tipo causa-efecto.

Las referencias a las carencias de capacitación y formación se extendieron a lo largo de todas las entrevistas, presentando su punto más elevado en los comentarios que se generaron al mostrarles la imagen de adultos en lo que parecía ser un taller relacionado con las TIC. El sentimiento positivo más reiterado fue acerca del valor de la interacción con otros, reiterándose el concepto de que es una de las principales acciones contribuyentes a limitar o eliminar los temores y miedos de los docentes. En sus expresiones, se muestran mucho más proclives a las actividades grupales aquellos docentes que aún no han pasado el "umbral" que les permite un trabajo autónomo o autodidacta con las TIC.

\subsection{La consideración de las TIC por parte de los docentes: herramienta y algo más}

La entidad de los sentimientos que se constatan al observar las expresiones docentes en relación al rol de las TIC nos lleva a pensar que dichas expresiones constituyen un indicador importante de sus creencias al respecto. Quienes presentan una actitud positiva ante las TIC, las consideran desde una herramienta necesaria, muy útil e incluso casi inevitable hasta una ayuda imprescindible que excede largamente su carácter de herramienta. En la "vereda" opuesta las consideraciones más "benignas" la consideran una herramienta más, llegando a expresiones extremistas que señalan insistentemente los posibles perjuicios que los llevan a considerarlas de utilidad relativa casi prescindibles en razón de las malas consecuencias que genera su uso. Al respecto, las fuentes académicas referidas señalan que si bien su carácter de herramienta es muy claro, su potencial va mucho más allá, afirmando que actualmente no es posible desarrollar una educación de calidad sin el concurso de TIC. Como tal, parten desde su integración a la actividad de enseñanza, hasta la generación de propuestas orientadas a la obtención de logros por parte de sus alumnos, incluyendo además de aspectos cognitivos el desarrollo de competencias asociadas. El reconocimiento del rol de las TIC no implica total aceptación y conformidad con los enfoques actuales. De hecho, se constatan reflexiones críticas, cuestionamientos e incluso dudas razonables y compartibles de parte de quienes demuestran formación en materia de TIC. Uno de los docentes que mostró mayor grado de integración de las TIC en sus prácticas manifiesta una preocupación que si bien no es mencionada por otros, figura entre los desafíos que señalan los referentes teóricos de la formación docente:

. lo que me preocupa es a largo plazo un escenario y es que los nuevos usuarios ... que pueden convertirse en docentes, no tengan el ... peso de una tradición cultural que los ayude a usar bien el soporte, ... es decir, ... estos que vayan a formar dentro de veinte años van a ser mucho más,... estar mucho más entrenados ... en el uso de las TIC ... seguramente, lo que no sé es si van a estar entrenados en el uso o en el consumo de las mismas..." 


\section{Conclusiones}

Las opiniones al igual que las acciones que fueron referidas por los docentes u observadas al investigador constituyen el reflejo de las actitudes consideradas, todas ellas posiblemente orientadas o influidas por sus creencias (dado el alcance de este estudio no es posible circunscribirlas inequívocamente). Si bien en parte se fundamentan en los aspectos racionales del pensamiento del profesor, también subyacen en ellas los sentimientos e incluso las emociones de cada docente, ya sea los más permanentes como también aquellos circunstanciales, incluyendo los que puedan generar inadvertidamente el propio investigador en su interacción con el educador.

Figura 6. Relación de creencias, actitudes y opiniones (en base a la representación gráfica de la "Teoría) de la acción razonada" de Ajzen y Fishbein (1988:194)

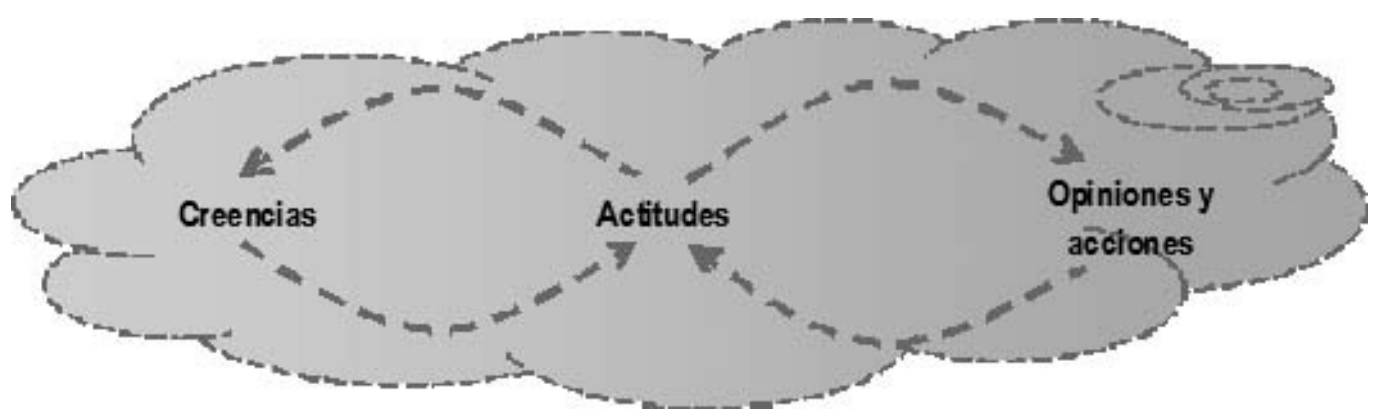

No constatamos "compartimentos estancos" entre opiniones, actitudes y creencias; se constituyen en una suerte de continuum, que más que ser explicado en clasificaciones taxativas debe ser interpretado de forma holística, para lo cual las técnicas cualitativas seleccionadas, si bien pueden ser optimizadas, fueron de gran ayuda (Fig.6).

\subsection{Actitudes constatadas}

Entre los aspectos a destacar se observaron actitudes hacia las TIC relacionadas a las interacciones entre docentes, el docente y sus alumnos y los alumnos entre sí. Entre ellas destacamos que la forma en que cada docente maneja el eventual desconocimiento en materia de empleo de TIC ante sus alumnos fue determinante (a nivel del contexto estudiado) de sus actitudes respecto a la integración de las TIC en sus prácticas educativas.

Asimismo se observó como claro indicador de la actitud general de cada docente hacia las TIC la forma en que los docentes consideran que las TIC influyen respecto a las tecnologías clásicas, en especial los libros.

Otro aspecto que constituyó un indicador de la actitud docente hacia las TIC fueron las referencias que realizaban en relación al alcance e impacto que atribuían a las TIC. Quienes son más proclives a la integración de las TIC en la educación manifestaron que su potencial incluye pero excede el carácter de herramienta, mientras que los docentes más críticos las consideraban como una herramienta más, que incluso puede generar perjuicios.

De forma coincidente con la literatura consultada se observó que la competencia de los docentes en materia de empleo de TIC constituye un aspecto significativo respecto a las actitudes hacia la integración de las tecnologías en sus prácticas. En relación a su influencia en las mencionadas actitudes se constataron grandes "umbrales" a partir de los cuales los docentes 
"miran diferente" a las TIC: un nivel inicial respecto al manejo de TIC, el cual es alcanzado por el docente al obtener acceso y sentirse seguro en cuanto al conocimiento y manejo básico de los programas y equipamientos informáticos, y un segundo nivel correspondiente a los docentes que se sienten seguros en el uso de las TIC al grado de que pueden manejarse en forma autónoma incluso con nuevos programas. Sin embargo, se constató que la capacitación de por sí no constituye un factor concluyente. De hecho, la orientación y la formación pedagógica de cada docente en cuanto al rol de las TIC parecerían tener mayor influencia que el dominio instrumental de las mismas.

\subsection{Posibles creencias}

En las actitudes anteriores se fueron esbozando las creencias subyacentes. Se percibe la existencia de creencias asociadas a la forma en que los docentes conciben la educación, entre ellas los enfoques centrados en la actividad de enseñanza del docente, o los enfoques centrados en los logros y motivaciones de sus alumnos. Estos aspectos se estima que influyen en varias de las actitudes mencionadas.

Profundizando en esta complejidad cabe recordar la heterogeneidad de visiones con que cada docente del instituto define lo que considera una "buena enseñanza" y, por extensión, base de lo que supone que constituye calidad educativa, así como la ausencia de menciones a las TIC. Tomamos estos aspectos como creencias dado que además de ser producto racional del pensamiento del profesor incluyen sentimientos forjados a lo largo de todo el desarrollo profesional del docente. Estos aspectos aparecen como coincidentes con las barreras internas o de segundo orden propias de las concepciones de cada docente acorde a como lo refiere la literatura consultada.

\subsection{Reflexiones personales}

Compartiendo los conceptos de los autores referidos en el marco teórico, constituye un reto superar el mero y básico empleo de la tecnología de forma de reproducir las prácticas tradicionales de enseñanza como simple sustituto de los libros. Es necesario repensar la capacitación y formación docentes incluyendo a las TIC en un rol que incluye, pero excede largamente su carácter de herramienta. El desafío para la capacitación parece centrarse en el desarrollo de las destrezas necesarias para manejar con confianza las TIC, mientras que para la formación lo constituiría permitir reflexionar en cuanto a las razones pedagógicas asociadas y derivadas de su uso, aspectos que en su conjunto constituyen la llave de su integración para una "buena enseñanza" y, por ende, parte integral de nuevos paradigmas de calidad educativa.

También consideramos vital avanzar de forma progresiva en la aceptación de aprender acerca de las tecnologías "de y junto a los alumnos", lo cual como docentes nos permitiría centrarnos en el irrenunciable e insustituible rol de orientadores epistemológicos, guiando el desarrollo de las competencias cognitivas de nuestros alumnos.
Podemos dejar para ellos el descubrimiento permanente de los "detalles" de la tecnología, contribuyendo a la motivación que significa para ellos sentirse funcionales y útiles a su propio desarrollo y el de los demás.

\subsection{Reflexiones sobre potenciales investigaciones}

En primera instancia podría ser de interés realizar otros estudios de caso para determinar en qué medida los hallazgos señalados pueden ser de aplicación en otras instituciones, en especial desde el punto de vista de la gestión educativa, y más específicamente en el desarrollo de proyectos que tengan como fin una mayor integración de tecnología en las prácticas docentes.

La falta de mención en el discurso, y por ende presumiblemente en el pensamiento de los docentes entrevistados en cuanto a la relación con los conocimientos pedagógicos, de 
contenidos y tecnológicos, lleva a considerar las dificultades que enfrentan en cuanto a poder lograr que sus alumnos magisteriales puedan generar reflexiones fundamentadas respecto a la contribución de las TIC para una "buena enseñanza". Este aspecto de por sí podría ser motivo de posteriores investigaciones que lo aborden, pudiendo incluir la adaptación y prueba del modelo que fuera tomado como base (Figura 7) y que de cierta forma nos permite resumir gráficamente el proceso de integración de las TIC en las prácticas docentes.

Figura 7. Proceso de integración de las TIC en las prácticas docentes y su relación con los diferentes tipos de conocimiento

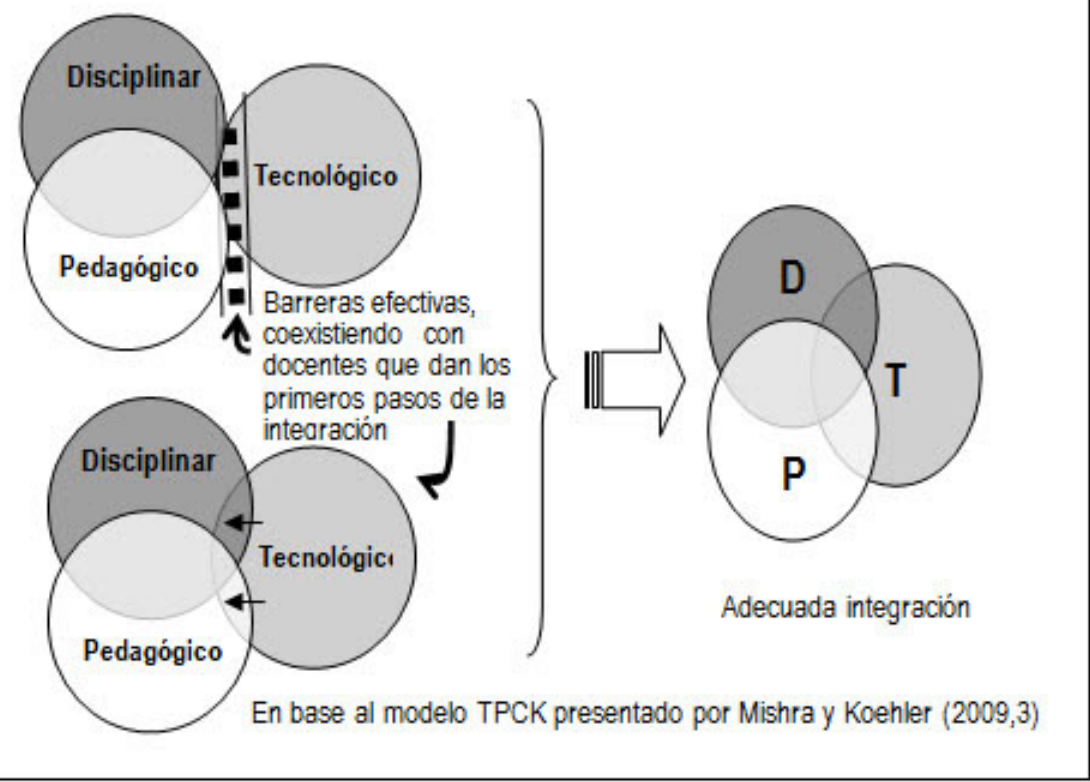

De forma complementaria, y pese a que no integra directamente la línea de investigación abordada sino más bien de otras asociadas a los diseños curriculares de la formación docente, se podría indagar acerca de la forma en que los planes de estudio vigentes contemplan esta aproximación entre los tres tipos de conocimiento mencionados.

En suma, y compartiendo las expresiones de uno de los docentes entrevistados y el sentir de muchos, sin que por ello se comprometa la reflexión que debe acompañar a la integración de las TIC en la educación en procura de una "buena enseñanza":

“...defiendo,... defendemos la alfabetización digital en las aulas, a capa y espada...”

Como reflexión o comentario final, pensamos que en el marco de la reformulación de los planes de capacitación y formación que señalan los expertos citados debería considerarse el desafío que representará para los institutos de formación docente, recibir y aprovechar el potencial que, de forma creciente e inevitable, traerán consigo las nuevas generaciones de inmigrantes digitales "criollos" producto del tiempo que les tocó vivir. 


\section{Bibliografía}

Ajzen, I.; Fishbein, M. (2001). The Influence of Attitudes on Behavior. Recuperado de: http:// web.psych.utoronto.ca/psy320/Required\%20readings_files/4-1.pdf

ANEP. (2011). Evaluación del Plan CEIBAL 2010. Documento resumen. Administración Nacional de Educación Pública (ANEP), Dirección Sectorial de Planificación Educativa (DSPE), Área de Evaluación del Plan CEIBAL. Recuperado de: http://www.anep.edu.uy/anepdata/0000031610. pdf

ANEP. (2011b). Programas de Plan 2008 para Magisterio. Administración Nacional de Educación Pública. Montevideo: ANEP.

Angeli, C.; Valanides, N. (2009). Epistemological and methodological issues for the conceptualization, development, and assessment of ICT-TPCK: Advances in technology and pedagogical content knowledge (TPCK). Computers and Education 52, 154-168. Recuperado de: http://teaching.cycu.edu.tw/pdf/2009_TPCK.pdf

Araya Umaña, S. (2002). Las representaciones sociales: ejes teóricos para su discusión. Cuaderno de ciencias sociales 127. Costa Rica: FLACSO (Facultad Latinoamericana de Ciencias Sociales).

Area Moreira, M. (2010). El proceso de integración y uso pedagógico de las TIC en los centros educativos. Un estudio de casos. Revista de Educación, 352 (mayo-agosto 2010), 77-97.

Ávalos, B. (2007). El desarrollo profesional continuo de los docentes: lo que nos dice la experiencia internacional y de la región latinoamericana. Revista Pensamiento Educativo, Vol.41 - Nro. 2 (diciembre 2007 "Formación inicial y continua de profesores"). Santiago de Chile: PUCC.

Bai, H.; Ertmer P. A. (2008). Teacher educators' beliefs and technology uses as predictors of preservice teachers' beliefs and technology attitudes. Journal of Technology and Teacher Education, 16(1), 93-112.

Boza, A. et al. (2010). Creencias del profesorado sobre el significado de la tecnología en la enseñanza: influencia para su inserción en los centros docentes andaluces. RELIEVE (Revista electrónica de investigación y evaluación educativa), v. 16, n. 1, recuperado de http://www. uv.es/RELIEVE/v16n1/RELIEVEv16n1_5.htm

Buckingham, D. (2008). Más allá de la tecnología: el aprendizaje infantil en la era de la cultura digital. Buenos Aires: Manantial.

Clark-Ibáñez, M. (2004). Framing the Social World With Photo-Elicitation Interviews. The American Behavioral Scientist, 47(12), 1507-1527.

Ertmer, P. A. (1999). Addressing first- and second-order barriers to change: Strategies for technology integration. Educational Technology Research and Development, 47(4), 47-61. Recuperado de: http://es.scribd.com/doc/45768935/null

ERTMER, P. A. (2005). Pedagogical beliefs: The final frontier in our quest for technology integration? Educational Technology Research and Development, 53(4), 25-39. Recuperado en: (en o de?) https://collaborate.education.purdue.edu/edci/ertmer/Docs/Conferences/ ETRD_5304.pdf

Gairín Sallán, J. (Coord.) et al. (2011). La Dirección de Centros Educativos en Iberoamérica. Reflexiones y Experiencias. Serie Informes - 2, Red AGE. Recuperado de: ftp://200.40.200.101/ libroredage.pdf 
Harper, D. (2002). Talking about pictures: a case for photo elicitation. Visual Studies, 17(1). Recuperado de: http://www.nyu.edu/classes/bkg/methods/harper.pdf

Litwin, E. (2005). Las configuraciones didácticas. Una nueva agenda para la enseñanza superior. Buenos Aires: Paidós.

Marcelo García, C. (1992). Cómo conocen los profesores la materia que enseñan. Algunas contribuciones de la investigación sobre conocimiento didáctico del contenido. Trabajo presentado en el Congreso "Las didácticas específicas en la formación del profesorado", Santiago, 6-10 de julio de 1992. Recuperado de: http://prometeo.us.es/ idea/miembros/01carlos-marcelo-garcia/archivos /Como\%20conocen.pdf

Marcelo García, C. (2001). Aprender a enseñar para la Sociedad del Conocimiento. Revista Complutense de Educación 12(2), 531-593. España: Univ. de Sevilla.

Merriam, S. (1998). Qualitative Research and Cases Study Applications in Education. San Francisco: Jossey-Bass Publishers.

Mishra, P.)Y Koehler, M.J. (2008). Introducing Technological Pedagogical Content Knowledge. Trabajo presentado en Annual Meeting of the American Educational Research Association, New York City, 24-28 de marzo de 2008. Recuperado de: http://punya.educ.msu.edu/presentations/ AERA2008/MishraKoehler_AERA2008.pdf

Morales Vallejo, P. (2011). Guía para construir cuestionarios y escalas de actitudes. Recuperado de: http://www.upcomillas.es/personal/peter/otrosdocumentos/ guiaparaconstruirescalasdeactitudes.pdf

Pajares, M. F. (1992). Teachers' Beliefs and Educational Research: Cleaning Up a Messy Construct. Review of Educational Research, 62(3), 307-332. Washington: American Educational Research Association.

Pérez Gómez, A. y Gimeno Sacristán, J. (1988). Pensamiento y acción en el profesor: de los estudios sobre la planificación al pensamiento práctico. Infancia y aprendizaje, 42, 37-64.

PLAN CEIBAL. (2009). Monitoreo y evaluación educativa del Plan CEIBAL. Primeros resultados a nivel nacional. Recuperado de: http://www.CEIBAL.org.uy/docs/ evaluacion_educativa_plan_ CEIBAL_resumen.pdf

PLAN CEIBAL. (2011). Segundo informe nacional de monitoreo y evaluación del Plan CEIBAL, 2010. Departamento de Monitoreo y Evaluación del Plan CEIBAL 2011. Recuperado de: http:// www.CEIBAL.org.uy/docs/Segundo-informe-nacional-de-monitoreo-y-evaluacion-del-PlanCEIBAL-2010.pdf

Prensky, M. (2001). Digital natives, digital inmigrants. Recuperado de: www.marcprensky.com Quinteros, M.del L. (2008). Buenas prácticas de enseñanza que incorporan el uso de TIC en Bachilleratos de Educación Secundaria. (Tesis de Maestría). Instituto de Educación, Universidad ORT Uruguay, Montevideo.

Rodríguez Zidán, E. Y Téliz F. (2011). Implementación del Plan CEIBAL en Uruguay: Revisión de Investigaciones y Desafíos de Mejora. Revista Iberoamericana de Evaluación Educativa, 4(2), 55-71. Recuperada de: http://www.rinace.net/riee/numeros/vol4-num2/art3.pdf

Sarabia, B. et al. (1992). Los contenidos en la reforma, enseñanza y aprendizaje de conceptos, procedimientos y actitudes. Madrid: Santillana. 
Schmidt, D.A. et al. (2009). Technological Pedagogical Content Knowledge (TPACK): The Development and Validation of an Assessment Instrument for Preservice Teachers. Journal of Research on Technology in Education, 42(2), 123-149. Recuperado de: www.eric.ed.gov/ ERICWebPortal/recordDetail?accno=EJ868626

Serrano Sánchez, R. (2010). Pensamientos del profesor: un acercamiento a las creencias y concepciones sobre el proceso de enseñanza-aprendizaje en la Educación Superior. Revista de Educación, 352 (mayo-agosto 2010), 267-287. Málaga: Universidad de Málaga.

Stake, R.E. (1998). Investigación con estudio de casos. Madrid: Morata

Tondeur, J.; Van Braak, J. Et al. (2008). Exploring the link between teachers' educational belief profiles and different types of computer use in the classroom.Computers in Human Behavior 24, 2541-2553. Recuperado de: http://www.sciencedirect.com/science/article/pii/ S0360131508000377

UNESCO. (2008). Estándares de competencias en TIC para docentes. Recuperado en: (en o de?) www.oei.es/tic/UNESCOEstandaresDocentes.pdf

Vaillant, D. (2007). Mejorando la formación y el desarrollo profesional docente en Latinoamérica. Revista Pensamiento Educativo, 41(2), 207-222.

Fecha de recibido: 14/02/2013

Fecha de aceptación: 30/05/2013

* Master en Educación, Universidad ORT Uruguay. Licenciado en Sistemas Navales, Escuela Naval. Analista Programador, Universidad ORT Uruguay. Docente, Jefe de Área Académica y Asesor en educación, Sistema Integral de Enseñanza Naval. 